Sharif University of Technology
Scientia Iranica
SCIENTIA
I RAN I CA
Thttp://scientiairanica.sharif.edu

\title{
Coupling of energy and harmonic balance methods for solving a conservative oscillator with strong odd-nonlinearity
}

\author{
Md. Abdur Razzak \\ Department of Mathematics, Rajshahi University of Engineering and Technology (RUET), Kazla, Rajshahi-6204, Bangladesh.
}

Received 5 September 2015; received in revised form 2 June 2016; accepted 28 January 2017

\author{
KEYWORDS \\ Nonlinear oscillation; \\ Odd-nonlinearity; \\ Cubic-quintic Duffing \\ oscillator; \\ Energy balance \\ method; \\ Harmonic balance \\ method.
}

\begin{abstract}
In this paper, a new analytical technique, i.e. a combination of the Energy Balance Method (EBM) with Harmonic Balance Method (HBM), is presented to obtain higher-order approximations of a conservative oscillator with strong odd nonlinearity. To show the accuracy of the present method, one nonlinear oscillator, named as cubic-quintic Duffing oscillator, is investigated. The results obtained in this paper are compared with those determined by other methods and exact solutions. The results give high accuracy and also provide better results than other existing results for both small and large amplitudes of oscillation. The main advantage of the present paper is its simplicity, which contains a few harmonic terms with lower order terms, and these terms make the solution converge quickly. The present technique can be used for other nonlinear oscillators.
\end{abstract}

(C) 2018 Sharif University of Technology. All rights reserved.

\section{Introduction}

The study of nonlinear oscillations is a necessary issue in engineering, physical science, applied mathematics, mechanical structures, nonlinear circuits, chemical oscillation, and many real-world applications [130]. Nonlinear oscillations are modeled by nonlinear differential equations. Many analytical techniques have been developed to solve these nonlinear differential equations. One of the most widely used techniques is perturbation method [1-4] whereby the nonlinearities are small. However, these techniques have many shortcomings and cannot be used due to strongly nonlinear systems. To overcome these shortcomings, many analytical techniques, such as variational iterative method $[5,6]$, homotopy perturbation

\footnotetext{
*. Tel.: +8801710441198

E-mail address: ra_m@ruet.ac.bd
}

method [7-9], iterative method [10-12], harmonic balance method [13-17], variational approach [18,19], and coupled method [20], are used to solve strongly nonlinear equations. The energy balance method [21,22] is also another technique to obtain a first-order approximation of strongly nonlinear oscillators. Usually, a set of algebraic equations with complex nonlinearities appears when EBM is formulated for determining higher-order approximations. On the contrary, some authors [23,24] have extended the energy balance method to obtain higher-order approximations, but the algebraic equations are not solved analytically.

The Duffing equation is a well-known nonlinear differential equation $[20,25,26]$ which is related to many practical engineering systems, such as the classical nonlinear spring system with odd nonlinear restoring characteristics [3], and has become applicable more recently in different physical phenomena [25]. There have been many variations of Duffing equation, for instance, the Duffing-harmonic equation [11,12] and the cubicquintic Duffing equation. The unperturbed cubic- 
quintic Duffing equation can be found in the modeling of the free vibration of a restrained uniform beam carrying an intermediate lumped mass and undergoing large amplitudes of oscillation in the unimodel Duffingtype temporal problem [26], the nonlinear dynamics of a slender elastica, the generalized Pochhammer-Chree (PC) equation, and the compound Korteweg-de Vries $(\mathrm{KdV})$ equation [26]. A differential equation with fifthpower nonlinearity is very difficult to handle due to the presence of strong nonlinearity.

Due to the presence of fifth-power nonlinearity, the accuracy of approximate analytical methods becomes extremely demanding [20]. Recently, several authors $[23,24]$ have extended the energy balance method to determine higher-order approximations. The limitation of the articles [23,24] is that they have not analytically solved algebraic equations; instead, they have only solved these algebraic equations numerically. On the other hand, Khan et al. [20] used coupled method of He's homotopy perturbation method [7] and variational formulation [18] to obtain higher-order approximations for nonlinear cubic-quintic Duffing equations. However, the first, second, even third-order approximations bring about unfavorable results, as compared with the exact solution. Furthermore, Guo et al. [27] obtained the analytical periodic solutions of the oscillator up to third-order approximation.

In this paper, a new analytical technique, combining the energy balance method [23] with harmonic balance method [17], has been presented to obtain higherorder approximations for nonlinear cubic-quintic Duffing equations. Generally, the second-order approximate frequency and the corresponding periodic solution have been determined containing a few harmonic terms with lower order terms. The algebraic equations are analytically solved in this paper easily. The secondorder approximate frequencies (obtained in this paper) show high accuracy in both small and large amplitudes of oscillation and also better than those obtained in [20] (calculated by the second-, third-, and fourth-order approximate frequencies). Moreover, compared to the other second-order approximation, the present method gives better results obtained by Guo et al. [27].

A cubic-quintic Duffing oscillator of a conservative autonomous system can be described by the following differential equation with cubic-quintic nonlinearities $[20,26,27]$ :

$$
\ddot{u}+\alpha u+\beta u^{3}+\gamma u^{5}=0,
$$

with initial conditions:

$$
u(0)=A, \quad \dot{u}(0)=0 .
$$

It is a simple harmonic oscillator if $\alpha \neq 0, \beta=\gamma=0$; it is a cubic Duffing oscillator if $\beta \neq 0, \gamma=0$; further, it is a quintic oscillator if $\gamma \neq 0, \beta=0$. Otherwise, it is a cubic-quintic oscillator if $\beta$ and $\gamma$ do not vanish (see $[20,26,27])$.

It should be noted that, in the case of $0<A<1$, system Eq. (1) will present small oscillations. On the other hand, in the case of $A \geq 1$, system Eq. (1) will present large oscillations (see [20,26,27]).

\section{The basic idea of He's energy balance method}

According to the energy balance method $[21,22,28,29]$, a variational principle for the oscillation is established, and then the corresponding Hamiltonian is considered from which the angular frequency can be easily founded by several residual methods.

Let us consider a general form of a nonlinear oscillator with initial conditions in the following form $[28,29]$ :

$$
\ddot{u}+f(u)=0, \quad u(0)=A, \quad \dot{u}(0)=0 .
$$

Its variational principle can be written as follows:

$$
J(u)=\int_{0}^{T / 4}\left[-\frac{1}{2} \dot{u}^{2}+F(u)\right] d t
$$

where $T=\frac{2 \pi}{\omega}$ is a period of nonlinear oscillation and $F(u)=\int f(u) d u$.

The Hamiltonian can be written as follows:

$$
H(t)=-\frac{1}{2} \dot{u}^{2}+F(u)=F(A) .
$$

Eq. (5) gives the following residual:

$$
R(t)=-\frac{1}{2} \dot{u}^{2}+F(u)-F(A)=0 .
$$

We consider the first-order approximate solution in the following form:

$$
u(t)=A \cos \omega t,
$$

Substituting Eq. (7) into Eq. (6) yields the following residual:

$$
R(t)=-\frac{1}{2} A^{2} \omega^{2} \sin ^{2} \omega t+F(A \cos \omega t)-F(A)=0
$$

Finally, collocation at $\omega t=\frac{\pi}{4}$ gives $[28,29]$ :

$$
\omega=\frac{2}{A} \sqrt{F(A)-F\left(\frac{\sqrt{2}}{2} A\right)} .
$$




\section{Application of the coupled energy and harmonic balance methods}

The variational principle of Eq. (1) can be written as follows:

$$
J(u)=\int_{0}^{T / 4}\left[-\frac{1}{2} \dot{u}^{2}+\alpha \frac{u^{2}}{2}+\beta \frac{u^{4}}{4}+\gamma \frac{u^{6}}{6}\right] d t .
$$

Its Hamiltonian, therefore, can be written in the following form:

$$
\begin{aligned}
H(u)= & \frac{\dot{u}^{2}}{2}+\frac{\alpha u^{2}}{2}+\frac{\beta u^{4}}{4}+\frac{\gamma u^{6}}{6}=\frac{\alpha A^{2}}{2} \\
& +\frac{\beta A^{4}}{4}+\frac{\gamma A^{6}}{6} .
\end{aligned}
$$

In order to obtain more accuracy, consider the secondorder approximate solution of Eq. (1) in the following form [17]:

$$
u(t)=A\left(\left(1-u_{3}\right) \cos \omega t+u \cos 3 \omega t\right) .
$$

Eq. (12) must satisfy initial conditions given in Eq. (2). In order to calculate the residual, by substituting Eq. (12) into Eq. (11), we obtain:

$$
\begin{aligned}
R(t)= & \frac{1}{2}\left(-\omega A\left(\left(1-u_{3}\right) \sin \omega t+3 \sin 3 \omega t\right)\right)^{2} \\
& \left.+\frac{\alpha}{2}\left(A\left(\left(1-u_{3}\right)\right) \cos \omega t+u \cos 3 \omega t\right)\right)^{2} \\
& \left.+\frac{\beta}{4}\left(A\left(\left(1-u_{3}\right)\right) \cos \omega t+u \cos 3 \omega t\right)\right)^{4} \\
& \left.+\frac{\gamma}{6}\left(A\left(\left(1-u_{3}\right)\right) \cos \omega t+u \cos 3 \omega t\right)\right)^{6} \\
& -\frac{\alpha A^{2}}{2}-\frac{\beta A^{4}}{4}-\frac{\gamma A^{6}}{6} .
\end{aligned}
$$

Now, through dividing Eq. (13) by factor $A^{2} \sec \omega t$ and then equating the coefficients of the terms $\cos \varphi$ and $\cos 3 \varphi$ from the integral:

$$
\int_{0}^{T / 4} \frac{R(t) \cos (2 n-1) \omega t}{A^{2} \sec \omega t} d t, \quad n=1,2 .
$$

Respective zeros are obtained as follows:

$$
\begin{aligned}
& \omega^{2}\left(1+4 u_{3}\right)-\alpha-4 u_{3} \alpha-3 A^{2} \beta / 4-5 A^{2} u_{3} \beta / 2 \\
& -29 A^{4} \gamma / 48-7 A^{4} u_{3} \gamma / 4+\cdots=0 \\
& \omega^{2}\left(-1+2 u_{3}\right)+\alpha+2 u_{3} \alpha+5 A^{2} \beta / 8+A^{2} u_{3} \beta / 2 \\
& +7 A^{4} \gamma / 16+\cdots=0 .
\end{aligned}
$$

It is noted that dividing factor $A^{2} \cos \omega t$ makes the solution converge rapidly and also gives significantly better results than other existing methods do.

For the first approximation, by setting $u_{3}=0$ in Eq. (15), the first approximate frequency is obtained as follows:

$$
48 \alpha+36 A^{2} \beta+29 A^{4} \gamma-48 \omega^{2}=0 .
$$

Solving Eq. (17) for $\omega$, the following is obtained:

$$
\omega=\omega_{1}(A)=\sqrt{\alpha+\frac{3 A^{2} \beta}{4}+\frac{29 A^{4} \gamma}{48}} .
$$

Eliminating $\omega$ from these two Eqs. (15) and (16), the equation for $u_{3}$ is obtained as follows:

$$
\begin{aligned}
& 1-\frac{3\left(32 \alpha+20 A^{2} \beta+15 A^{4} \gamma\right)}{A^{2}\left(3 \beta+4 A^{2} \gamma\right)} u_{3}+24 u_{3}^{2}-96 u_{3}^{3} \\
& +\cdots=0
\end{aligned}
$$

Eq. (19) can be written as follows:

$$
u_{3}=\mu\left(1+24 u_{3}^{2}-96 u_{3}^{3}+\cdots\right),
$$

where:

$$
\mu=\frac{A^{2}\left(3 \beta+4 A^{2} \gamma\right)}{3\left(32 \alpha+20 A^{2} \beta+15 A^{4} \gamma\right)} .
$$

Now, $u_{3}$ can be obtained in powers of $\mu$ of the form $u_{3}=l_{1} \mu+l_{2} \mu^{2}+l_{3} \mu^{3}+\ldots$ (see [17] for details) where unknown coefficients $l_{1}, l_{2}, l_{3}, \ldots$ are to be determined. Therefore, we have obtained the solution of Eq. (20) as follows:

$$
u_{3}=\mu+24 \mu^{3}-96 \mu^{4}+\cdots .
$$

It is noted that the series of $u_{3}$ converge to all values of $A$.

By solving Eq. (15) for $\omega$, the second approximate frequency is obtained as follows:

$$
\begin{aligned}
\omega & =\omega_{2}(A)= \\
& \sqrt{\frac{\alpha+4 u_{3} \alpha+\frac{3 A^{2} \beta}{4}+\frac{5 A^{2} u_{3} \beta}{2}+\frac{29 A^{4} \gamma}{48}+\frac{7 A^{4} u_{3} \gamma}{4}}{1+u_{3}}},
\end{aligned}
$$

where $u_{3}$ is given in Eq. (21).

Therefore, the second-order approximation becomes:

$$
\left.u(t)=A\left(\left(1-u_{3}\right)\right) \cos \omega t+u_{3} \cos 3 \omega t\right)
$$

where $u_{3}$ and $\omega$ are given in Eqs. (21) and (22), respectively. 


\section{Results and discussion}

A new analytical technique coupled by the energy and harmonic balance methods has been presented to determine the approximate frequency and the corresponding solution to cubic-quintic Duffing oscillator. The method is valid for both small $(0<A<1)$ and large $(A \geq 1)$ amplitudes of oscillation. Recently, Khan et al. $[20,27]$ have investigated the same oscillator by coupling homotopy with variational approaches and obtained the first-, second-, third-, and forth-order approximate frequencies. However, the determination of the third- and fourth-order approximations is a laborious process. In this situation, the determination of (first-order (given in Eq. (18) and second-order (given in Eq. (22)) approximations obtained in this paper is an easy and straightforward process.

To verify the efficiency and accuracy of the present method for cubic-quintic Duffing oscillator, in comparison with other results and the exact result, three cases are given: $\alpha=\beta=\gamma=1, \alpha=5, \beta=3$, $\gamma=1$ and $\alpha=1, \beta=10, \gamma=100$ (see [20]). The relative errors of frequencies are defined as follows [26]:

$$
\text { Error }(\%)=\frac{\left|\omega_{i}-\omega_{\text {Exact }}\right|}{\omega_{\text {Exact }}}, i=1,2,3,4, \cdots
$$

The relative errors of the first- and second-order analytical approximations obtained in this paper are compared with the exact solution, providing results less than $4.077 \%$ and $0.102 \%$, respectively, in the case of $A \geq 1$ (i.e., large amplitudes). In Tables 1-3, the relative errors for the approximate frequencies of different parameters are presented.

On the other hand, the relative errors of the first-, second-, third-, and fourth-order analytical approximations obtained by [20] are compared with the exact solution, providing results less than $25.149 \%, 15.519 \%$, $7.050 \%$, and $0.154 \%$, respectively.

Furthermore, the relative errors of the secondorder analytical approximations obtained by [27] are compared with the exact solution which are less than $1.078 \%$.

Based on these three tables, we also see that the present method gives better results than those obtained in $[20,27]$ for small values of amplitude, $0<A<1$. The convergence rate of the present method is faster

Table 1. Comparison of the present frequency with existing results for cubic-quintic Duffing oscillator when $\alpha=\beta=\gamma=1$.

\begin{tabular}{|c|c|c|c|c|c|c|c|c|}
\hline \multirow[b]{2}{*}{$\boldsymbol{A}$} & \multirow[b]{2}{*}{$\omega_{e}$} & \multicolumn{3}{|c|}{ Khan et al. [20] } & \multicolumn{2}{|c|}{ Guo et al. [27] } & \multicolumn{2}{|c|}{ Present study } \\
\hline & & $\begin{array}{c}\omega_{1} \\
\operatorname{Er}(\%)^{*}\end{array}$ & $\begin{array}{c}\omega_{2} \\
\operatorname{Er}(\%)\end{array}$ & $\begin{array}{c}\omega_{3} \\
\operatorname{Er}(\%)\end{array}$ & $\begin{array}{c}\omega_{4} \\
\operatorname{Er}(\%)\end{array}$ & $\begin{array}{c}\omega_{2} \\
\operatorname{Er}(\%)\end{array}$ & $\begin{array}{c}\omega_{1} \\
\operatorname{Er}(\%)\end{array}$ & $\begin{array}{c}\omega_{2} \\
\operatorname{Er}(\%)\end{array}$ \\
\hline 0.1 & 1.0037770 & $\begin{array}{c}1.0025125 \\
0.126\end{array}$ & $\begin{array}{c}1.0028276 \\
0.095\end{array}$ & $\begin{array}{c}1.0031009 \\
0.067\end{array}$ & $\begin{array}{c}1.0034276 \\
0.035\end{array}$ & $\begin{array}{c}1.0037730 \\
0.000\end{array}$ & $\begin{array}{c}1.0037732 \\
0.000\end{array}$ & $\begin{array}{c}1.0037722 \\
0.000\end{array}$ \\
\hline 0.5 & 1.1065487 & $\begin{array}{c}1.0698277 \\
3.319\end{array}$ & $\begin{array}{c}1.0792589 \\
2.467\end{array}$ & $\begin{array}{c}1.0877056 \\
1.703\end{array}$ & $\begin{array}{c}1.0974873 \\
0.819\end{array}$ & $\begin{array}{c}1.1065755 \\
0.003\end{array}$ & $\begin{array}{c}1.1069148 \\
0.033\end{array}$ & $\begin{array}{c}1.1062745 \\
0.025\end{array}$ \\
\hline 1 & 1.5235914 & $\begin{array}{c}1.3462912 \\
11.637\end{array}$ & $\begin{array}{c}1.3984287 \\
8.215\end{array}$ & $\begin{array}{c}1.4456576 \\
5.115\end{array}$ & $\begin{array}{c}1.4951413 \\
1.867\end{array}$ & $\begin{array}{c}1.5250736 \\
0.097\end{array}$ & $\begin{array}{c}1.5343294 \\
0.705\end{array}$ & $\begin{array}{c}1.5224068 \\
0.078\end{array}$ \\
\hline 5 & 19.1815720 & $\begin{array}{c}14.4503460 \\
24.666\end{array}$ & $\begin{array}{c}16.2514248 \\
15.276\end{array}$ & $\begin{array}{c}17.8276787 \\
7.058\end{array}$ & $\begin{array}{c}19.1806374 \\
0.005\end{array}$ & $\begin{array}{c}19.3735477 \\
1.001\end{array}$ & $\begin{array}{c}19.9337444 \\
3.921\end{array}$ & $\begin{array}{c}19.1676301 \\
0.073\end{array}$ \\
\hline 10 & 75.1776276 & $\begin{array}{c}56.3560104 \\
25.036\end{array}$ & $\begin{array}{c}63.5547600 \\
15.461\end{array}$ & $\begin{array}{c}69.8760834 \\
7.052\end{array}$ & $\begin{array}{c}75.2651825 \\
0.115\end{array}$ & $\begin{array}{c}75.9737510 \\
1.060\end{array}$ & $\begin{array}{c}78.2155142 \\
4.041\end{array}$ & $\begin{array}{c}75.1074146 \\
0.093\end{array}$ \\
\hline 20 & 299.22427 & $\begin{array}{c}224.05580 \\
25.121\end{array}$ & $\begin{array}{c}252.83170 \\
15.504\end{array}$ & $\begin{array}{c}278.12708 \\
7.051\end{array}$ & $\begin{array}{c}299.65771 \\
0.145\end{array}$ & $\begin{array}{c}302.43540 \\
1.073\end{array}$ & $\begin{array}{c}311.39632 \\
4.068\end{array}$ & $\begin{array}{c}298.92664 \\
0.099\end{array}$ \\
\hline 50 & 1867.5796 & $\begin{array}{c}1397.9900 \\
25.145\end{array}$ & $\begin{array}{c}1577.7996 \\
15.516\end{array}$ & $\begin{array}{c}1735.9103 \\
7.050\end{array}$ & $\begin{array}{c}1870.4300 \\
0.153\end{array}$ & $\begin{array}{c}1887.6949 \\
1.077\end{array}$ & $\begin{array}{c}1943.6866 \\
4.075\end{array}$ & $\begin{array}{c}1865.6890 \\
0.101\end{array}$ \\
\hline 100 & 7468.8525 & $\begin{array}{c}5590.6172 \\
25.148\end{array}$ & $\begin{array}{c}6309.8315 \\
15.518\end{array}$ & $\begin{array}{c}6942.2827 \\
7.050\end{array}$ & $\begin{array}{c}7480.3364 \\
0.154\end{array}$ & $\begin{array}{c}7549.3401 \\
1.078\end{array}$ & $\begin{array}{c}7773.2984 \\
4.076\end{array}$ & $\begin{array}{c}7461.2727 \\
0.102\end{array}$ \\
\hline 500 & 186709.59 & $\begin{array}{c}139754.70 \\
25.149\end{array}$ & $\begin{array}{c}157734.86 \\
15.519\end{array}$ & $\begin{array}{c}173546.20 \\
7.050\end{array}$ & $\begin{array}{c}186997.34 \\
0.154\end{array}$ & $\begin{array}{c}188721.99 \\
1.078\end{array}$ & $\begin{array}{c}194320.88 \\
4.077\end{array}$ & $\begin{array}{c}186519.96 \\
0.102\end{array}$ \\
\hline 1000 & 746836.94 & $\begin{array}{c}559017.44 \\
25.149\end{array}$ & $\begin{array}{c}630938.13 \\
15.519\end{array}$ & $\begin{array}{c}694183.44 \\
7.050\end{array}$ & $\begin{array}{c}747988.00 \\
0.154\end{array}$ & $\begin{array}{c}754886.53 \\
1.078\end{array}$ & $\begin{array}{c}777282.07 \\
4.077\end{array}$ & $\begin{array}{c}746078.35 \\
0.102\end{array}$ \\
\hline
\end{tabular}

*: $\operatorname{Er}(\%)$ denotes the absolute percentage error. 
Table 2. Comparison of the present frequency with existing results for cubic-quintic Duffing oscillator when $\alpha=5$, $\beta=3$, and $\gamma=1$.

\begin{tabular}{|c|c|c|c|c|c|c|c|c|}
\hline \multirow[t]{2}{*}{$A$} & \multirow[t]{2}{*}{$\omega_{e}$} & \multicolumn{3}{|c|}{ Khan et al. [20] } & \multicolumn{2}{|c|}{ Guo et al. [27] } & \multicolumn{2}{|c|}{ Present study } \\
\hline & & $\begin{array}{c}\omega_{1} \\
\operatorname{Er}(\%)^{*}\end{array}$ & $\begin{array}{c}\omega_{2} \\
\operatorname{Er}(\%)\end{array}$ & $\begin{array}{c}\omega_{3} \\
\operatorname{Er}(\%)\end{array}$ & $\begin{array}{c}\omega_{4} \\
\operatorname{Er}(\%)\end{array}$ & $\begin{array}{c}\omega_{2} \\
\operatorname{Er}(\%)\end{array}$ & $\begin{array}{c}\omega_{1} \\
\operatorname{Er}(\%)\end{array}$ & $\begin{array}{c}\omega_{2} \\
\operatorname{Er}(\%)\end{array}$ \\
\hline 0.1 & 2.2411156 & $\begin{array}{c}2.2394266 \\
0.075\end{array}$ & $\begin{array}{c}2.2398469 \\
0.057\end{array}$ & $\begin{array}{c}2.2402105 \\
0.041\end{array}$ & $\begin{array}{c}2.2406456 \\
0.021\end{array}$ & $\begin{array}{c}2.2411070 \\
0.001\end{array}$ & $\begin{array}{c}2.2411070 \\
0.000\end{array}$ & $\begin{array}{c}2.2411063 \\
0.000\end{array}$ \\
\hline 0.5 & 2.3661575 & $\begin{array}{c}2.3226130 \\
1.840\end{array}$ & $\begin{array}{c}2.3337476 \\
1.370\end{array}$ & $\begin{array}{c}2.3434565 \\
0.960\end{array}$ & $\begin{array}{c}2.3548751 \\
0.477\end{array}$ & $\begin{array}{c}2.3661560 \\
0.014\end{array}$ & $\begin{array}{c}2.3664870 \\
0.003\end{array}$ & 2.3660775 \\
\hline 1 & 2.7962794 & $\begin{array}{c}2.6100767 \\
6.659\end{array}$ & $\begin{array}{c}2.6612775 \\
4.828\end{array}$ & $\begin{array}{c}2.7063482 \\
3.216\end{array}$ & $\begin{array}{c}2.7566507 \\
1.417\end{array}$ & $\begin{array}{c}2.7966959 \\
0.015\end{array}$ & $\begin{array}{c}2.8025286 \\
0.223\end{array}$ & $\begin{array}{c}2.7958356 \\
0.016\end{array}$ \\
\hline 5 & 20.2164536 & $\begin{array}{c}15.4211702 \\
23.720\end{array}$ & $\begin{array}{c}17.2236977 \\
14.804\end{array}$ & $\begin{array}{c}18.7895069 \\
7.058\end{array}$ & $\begin{array}{c}20.1565380 \\
0.296\end{array}$ & $\begin{array}{c}20.3911022 \\
0.864\end{array}$ & $\begin{array}{c}20.9488464 \\
3.623\end{array}$ & $\begin{array}{c}20.2109616 \\
0.027\end{array}$ \\
\hline 10 & 76.1700134 & $\begin{array}{c}57.2712860 \\
24.811\end{array}$ & $\begin{array}{c}64.4817429 \\
15.345\end{array}$ & $\begin{array}{c}70.7962723 \\
7.055\end{array}$ & $\begin{array}{c}76.2022247 \\
0.042\end{array}$ & $\begin{array}{c}76.9486769 \\
1.022\end{array}$ & $\begin{array}{c}79.1938550 \\
3.970\end{array}$ & $\begin{array}{c}76.1106133 \\
0.078\end{array}$ \\
\hline 50 & 1868.5568 & $\begin{array}{c}1398.8853 \\
25.136\end{array}$ & $\begin{array}{c}1578.7106 \\
15.512\end{array}$ & $\begin{array}{c}1736.8159 \\
7.051\end{array}$ & $\begin{array}{c}1871.3540 \\
0.150\end{array}$ & $\begin{array}{c}1888.6547 \\
1.076\end{array}$ & $\begin{array}{c}1944.6521 \\
4.073\end{array}$ & $\begin{array}{c}1866.6778 \\
0.101\end{array}$ \\
\hline 100 & 7469.8296 & $\begin{array}{c}5591.5117 \\
25.146\end{array}$ & $\begin{array}{c}6310.7422 \\
15.517\end{array}$ & $\begin{array}{c}6943.1880 \\
7.050\end{array}$ & $\begin{array}{c}7481.2598 \\
0.153\end{array}$ & $\begin{array}{c}7550.2994 \\
1.078\end{array}$ & $\begin{array}{c}7774.2634 \\
4.076\end{array}$ & $\begin{array}{c}7462.2611 \\
0.101\end{array}$ \\
\hline 500 & 186710.58 & $\begin{array}{c}139755.59 \\
25.149\end{array}$ & $\begin{array}{c}157735.78 \\
15.519\end{array}$ & $\begin{array}{c}173547.11 \\
7.050\end{array}$ & $\begin{array}{c}186998.27 \\
0.154\end{array}$ & $\begin{array}{c}188722.95 \\
1.078\end{array}$ & $\begin{array}{c}194321.85 \\
4.077\end{array}$ & $\begin{array}{c}186520.95 \\
0.102\end{array}$ \\
\hline 1000 & 746837.94 & $\begin{array}{c}559018.31 \\
25.149\end{array}$ & $\begin{array}{c}630939.00 \\
15.519\end{array}$ & $\begin{array}{c}694184.38 \\
7.050\end{array}$ & $\begin{array}{c}747988.88 \\
0.154\end{array}$ & $\begin{array}{c}754887.48 \\
1.078\end{array}$ & $\begin{array}{c}777283.04 \\
4.077\end{array}$ & $\begin{array}{c}746079.34 \\
0.102\end{array}$ \\
\hline
\end{tabular}

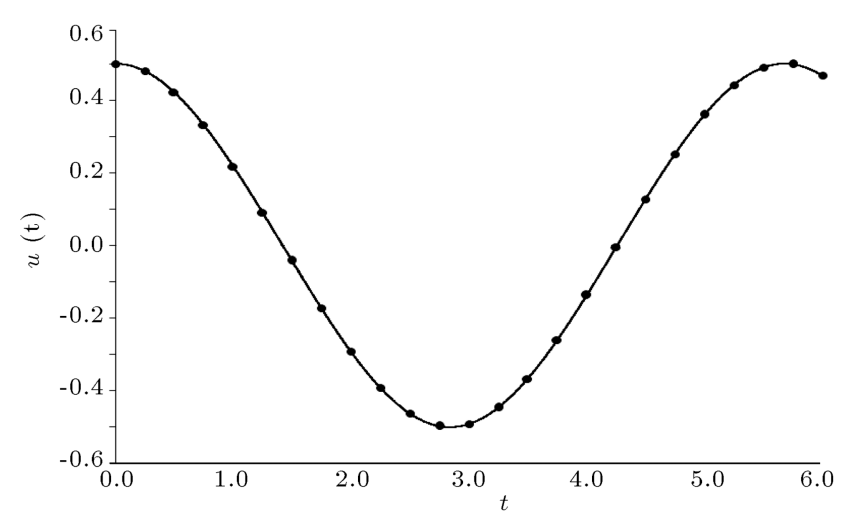

Figure 1. Comparison of approximate periodic solution obtained by the present method (denoted by circles) with the numerical solution obtained by the fourth-order Runge-Kutta method (denoted by solid line) for the cubic-quintic Duffing oscillator (Eq. (1)) for $\alpha=1, \beta=1$, $\gamma=1$, and $A=0.5)$.

than $[20,27]$. Therefore, the present method is suitable for solving Eq. (1), as compared to [20,27].

Furthermore, we have determined the secondorder approximate solutions to Eq. (1) for different

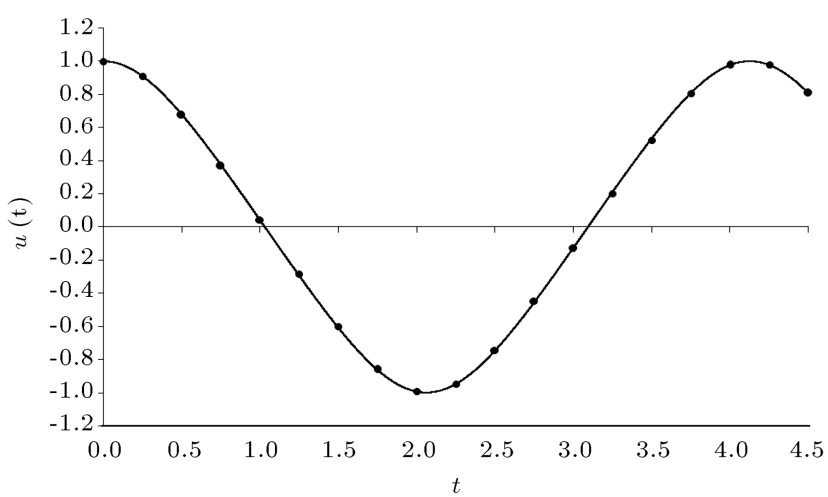

Figure 2. Comparison of approximate periodic solution obtained by the present method (denoted by circles) with the numerical solution obtained by the fourth-order Runge-Kutta method (denoted by solid line) for the cubic-quintic Duffing oscillator (Eq. (1)) for $\alpha=1, \beta=1$, $\gamma=1$, and $A=1.0$ ).

values of parameters $A, \alpha, \beta$, and $\gamma$, including all results with the corresponding numerical solutions obtained by fourth-order Runge-Kutta method. All results are presented in Figures 1-6. Based on these figures, we 
Table 3. Comparison of the present frequency with existing results for cubic-quintic Duffing oscillator when $\alpha=1, \beta=10$, and $\gamma=100$.

\begin{tabular}{|c|c|c|c|c|c|c|c|c|}
\hline \multirow[t]{2}{*}{$A$} & \multirow[t]{2}{*}{$\omega_{e}$} & \multicolumn{3}{|c|}{ Khan et al. [20] } & \multicolumn{2}{|c|}{ Guo et al. [27] } & \multicolumn{2}{|c|}{ Present study } \\
\hline & & $\begin{array}{c}\omega_{1} \\
\operatorname{Er}(\%)^{*}\end{array}$ & $\begin{array}{c}\omega_{2} \\
\operatorname{Er}(\%)\end{array}$ & $\begin{array}{c}\omega_{3} \\
\operatorname{Er}(\%)\end{array}$ & $\begin{array}{c}\omega_{4} \\
\operatorname{Er}(\%)\end{array}$ & $\begin{array}{c}\omega_{2} \\
\operatorname{Er}(\%)\end{array}$ & $\begin{array}{c}\omega_{1} \\
\operatorname{Er}(\%)\end{array}$ & $\begin{array}{c}\omega_{2} \\
\operatorname{Er}(\%)\end{array}$ \\
\hline 0.1 & 1.0397019 & $\begin{array}{c}1.0262188 \\
1.297\end{array}$ & $\begin{array}{c}1.0296117 \\
0.971\end{array}$ & $\begin{array}{c}1.0325994 \\
0.683\end{array}$ & $\begin{array}{c}1.0361322 \\
0.343\end{array}$ & $\begin{array}{c}1.0397000 \\
0.000\end{array}$ & $\begin{array}{c}1.0397315 \\
0.003\end{array}$ & $\begin{array}{c}1.0396423 \\
0.006\end{array}$ \\
\hline 0.5 & 2.5247023 & $\begin{array}{c}2.0501525 \\
18.796\end{array}$ & $\begin{array}{c}2.2114758 \\
12.407\end{array}$ & $\begin{array}{c}2.3542032 \\
6.753\end{array}$ & $\begin{array}{c}2.4890764 \\
1.411\end{array}$ & $\begin{array}{c}2.5350468 \\
0.410\end{array}$ & $\begin{array}{c}2.5789614 \\
2.150\end{array}$ & $\begin{array}{c}2.5236147 \\
0.043\end{array}$ \\
\hline 1 & 8.0100698 & $\begin{array}{c}6.1032777 \\
23.805\end{array}$ & $\begin{array}{c}6.8193183 \\
14.866\end{array}$ & $\begin{array}{c}7.4440041 \\
7.067\end{array}$ & $\begin{array}{c}7.9883609 \\
0.271\end{array}$ & $\begin{array}{c}8.0806905 \\
0.882\end{array}$ & $\begin{array}{c}8.3016063 \\
3.640\end{array}$ & $\begin{array}{c}8.0064163 \\
0.046\end{array}$ \\
\hline 5 & 187.19966 & $\begin{array}{c}140.20432 \\
25.105\end{array}$ & $\begin{array}{c}158.19193 \\
15.496\end{array}$ & $\begin{array}{c}174.00040 \\
7.051\end{array}$ & $\begin{array}{c}187.46034 \\
0.139\end{array}$ & $\begin{array}{c}189.20333 \\
1.070\end{array}$ & $\begin{array}{c}194.80482 \\
4.063\end{array}$ & $\begin{array}{c}187.01580 \\
0.098\end{array}$ \\
\hline 10 & 747.32526 & $\begin{array}{c}559.46490 \\
25.138\end{array}$ & $\begin{array}{c}631.39343 \\
15.513\end{array}$ & $\begin{array}{c}694.63605 \\
7.050\end{array}$ & $\begin{array}{c}748.44946 \\
0.151\end{array}$ & $\begin{array}{c}755.36618 \\
1.076\end{array}$ & $\begin{array}{c}777.76453 \\
4.073\end{array}$ & $\begin{array}{c}746.57252 \\
0.100\end{array}$ \\
\hline 50 & 18671.400 & $\begin{array}{c}13975.872 \\
25.148\end{array}$ & $\begin{array}{c}15773.896 \\
15.519\end{array}$ & $\begin{array}{c}17355.027 \\
7.050\end{array}$ & $\begin{array}{c}18700.148 \\
0.154\end{array}$ & $\begin{array}{c}18872.631 \\
1.078\end{array}$ & $\begin{array}{c}19432.522 \\
4.075\end{array}$ & $\begin{array}{c}18652.440 \\
0.102\end{array}$ \\
\hline 100 & 74684.133 & $\begin{array}{c}55902.148 \\
25.149\end{array}$ & $\begin{array}{c}63094.219 \\
15.519\end{array}$ & $\begin{array}{c}69418.750 \\
7.050\end{array}$ & $\begin{array}{c}74799.211 \\
0.154\end{array}$ & $\begin{array}{c}75489.084 \\
1.078\end{array}$ & $\begin{array}{c}77728.641 \\
4.077\end{array}$ & $\begin{array}{c}74608.280 \\
0.102\end{array}$ \\
\hline 500 & 1867091.6 & $\begin{array}{c}1397542.9 \\
25.149\end{array}$ & $\begin{array}{c}1577344.5 \\
15.519\end{array}$ & $\begin{array}{c}1735457.9 \\
7.050\end{array}$ & $\begin{array}{c}1869969.3 \\
0.154\end{array}$ & $\begin{array}{c}1887215.6 \\
1.078\end{array}$ & $\begin{array}{c}1943200.0 \\
4.076\end{array}$ & $\begin{array}{c}1865200.0 \\
0.101\end{array}$ \\
\hline 1000 & 7468365.0 & $\begin{array}{c}5590170.5 \\
25.149\end{array}$ & $\begin{array}{c}6309377.0 \\
15.519 \\
\end{array}$ & $\begin{array}{c}6941830.5 \\
7.050\end{array}$ & $\begin{array}{c}7479875.5 \\
0.154\end{array}$ & $\begin{array}{c}7548860.9 \\
1.078\end{array}$ & $\begin{array}{c}7772820.0 \\
4.077\end{array}$ & $\begin{array}{c}7460780.0 \\
0.102\end{array}$ \\
\hline
\end{tabular}

*: $\operatorname{Er}(\%)$ denotes the absolute percentage error.

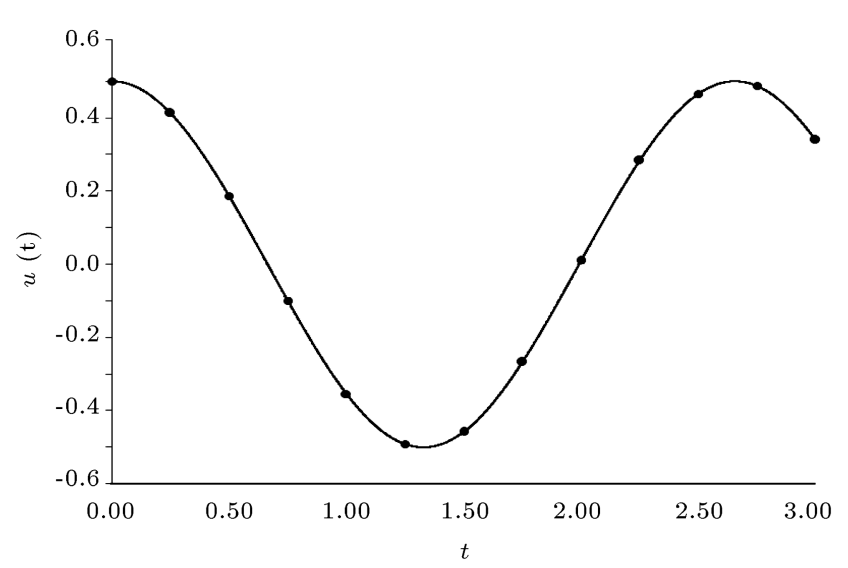

Figure 3. Comparison of approximate periodic solution obtained by the present method (denoted by circles) with the numerical solution obtained by the fourth-order Runge-Kutta method (denoted by solid line) for the cubic-quintic Duffing oscillator (Eq. (1)) for $\alpha=5, \beta=3$, $\gamma=1$, and $A=0.5$ ).

see that the present method's solutions are nicely in agreement with the corresponding numerical results for all values of parameters $A, \alpha, \beta$, and $\gamma$.

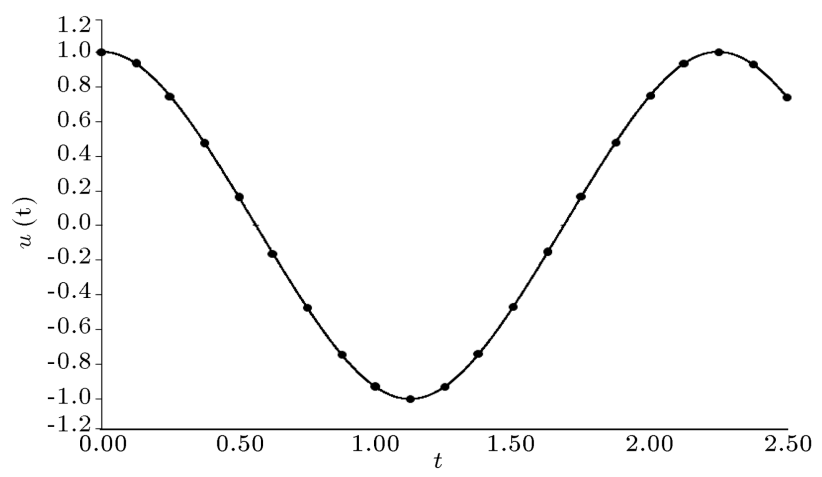

Figure 4. Comparison of approximate periodic solution obtained by the present method (denoted by circles) with the numerical solution obtained by the fourth-order Runge-Kutta method (denoted by solid line) for the cubic-quintic Duffing oscillator (Eq. (1)) for $\alpha=5, \beta=3$, $\gamma=1$, and $A=1.0$.

\section{Conclusion}

In this paper, a new simple analytical technique coupled by energy and harmonic balance methods was presented to solve the cubic-quintic Duffing oscillator. Next, The second-order approximation was 


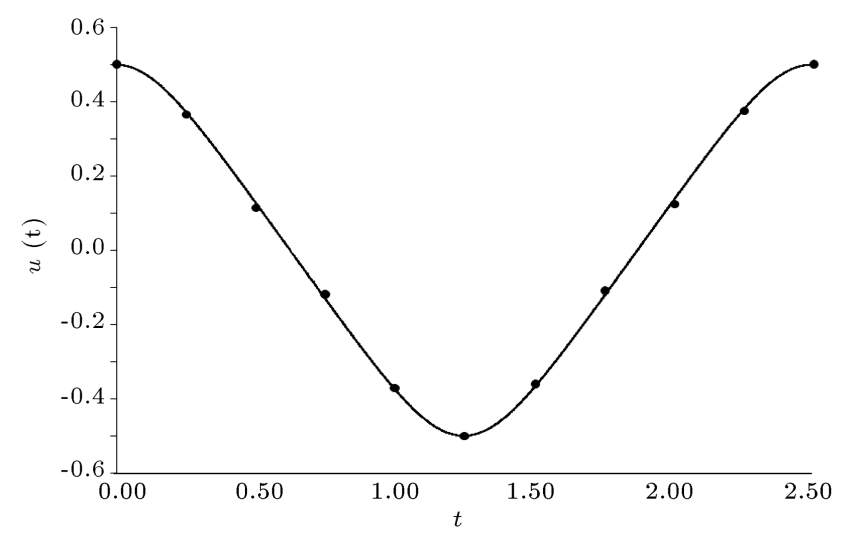

Figure 5. Comparison of approximate periodic solution obtained by the present method (denoted by circles) with the numerical solution obtained by the fourth-order Runge-Kutta method (denoted by solid line) for the cubic-quintic Duffing oscillator (Eq. (1)) for $\alpha=1$, $\beta=10, \gamma=100$, and $A=0.5$.

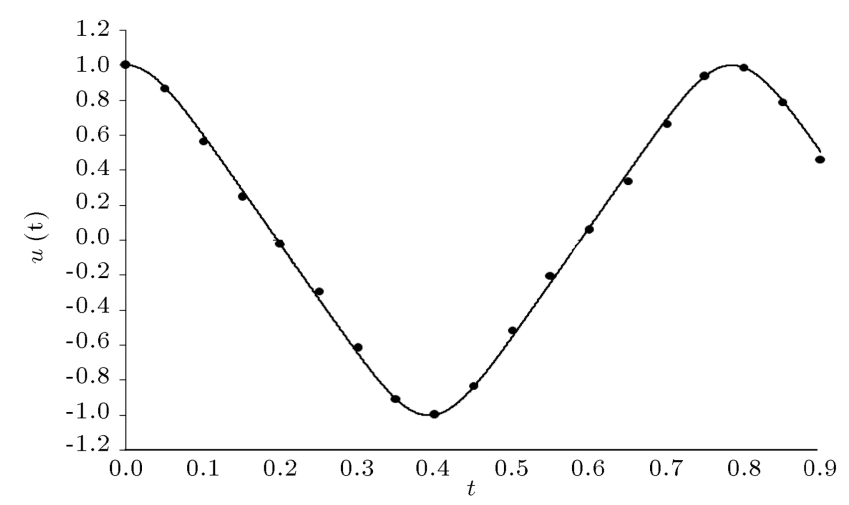

Figure 6. Comparison of approximate periodic solution obtained by the present method (denoted by circles) with the numerical solution obtained by the fourth-order Runge-Kutta method (denoted by solid line) for the cubic-quintic Duffing oscillator (Eq. (1)) for $\alpha=1$, $\beta=10, \gamma=100$, and $A=1.0)$.

determined. The solution contains a few harmonic terms and also a lower-order term. These terms make the solution converge rapidly. It was observed that the present method gives better results than other existing results do, for both small and large amplitudes of oscillation. It was proved that the present method is very effective, convenient and also gives more precise accuracy for solving strongly nonlinear oscillators.

\section{References}

1. Krylov, N.N. and Bogoliubov, N.N., Introduction to Nonlinear Mechanics, Princeton University Press, New Jersey (1947).

2. Bogoliubov, N.N. and Mitropolskii, Yu.A., Asymptotic Methods in the Theory of Nonlinear Oscillations, Gordan and Breach, New York (1961).
3. Nayfeh, A.H. and Mook, D.T., Nonlinear Oscillations, John Wiley \& Sons, New York (1979).

4. Nayfeh, A.H., Introduction to Perturbation Techniques, John Wiley \& Sons, New York (1981).

5. He, J.H., Wu, G.C., and Austin, F. "The variational iterative method which should be followed", Nonlinear Science Letter A, 1(1), pp. 1-30 (2010).

6. Herisanu, N. and Marinca, V. "A modified variational iterative method for strongly nonlinear oscillators", Nonlinear Science Letter A, 1(2), pp. 183-192 (2010).

7. He, J.H. "The homotopy perturbation method for nonlinear oscillators with discontinuous", Applied Mathematics and Computation, 151, pp. 287-292 (2004).

8. Ganji, D.D. "The application of He's homotopy perturbation method to nonlinear equations arising in heat transfer", Physics Letter A, 355, pp. 337-341 (2006).

9. Rafei, M., Ganji, D.D., and Daniali, H. "Solution of the epidemic model by homotopy perturbation method", Applied Mathematics and Computation, 187, pp. 10561062 (2007).

10. Mickens, R.E. "A general procedure for calculating approximation to periodic solutions of truly nonlinear oscillators", Journal of Sound and Vibration, 287, pp. 1045-1051 (2005).

11. Lim, C.W., Wu, B.S., and Sun, W.P. "Higher accuracy analytical approximations to the Duffing-harmonic oscillator", Journal of Sound and Vibration, 296, pp. 1039-1045 (2006).

12. Hu, H. "Solutions of Duffing-harmonic oscillator by an iteration procedure", Journal of Sound and Vibration, 298, pp. 446-452 (2006).

13. Mickens, R.E., Truly Nonlinear Oscillations, World Scientific, Singapore (2010).

14. Haque, B.M. I., Alam, M.S., and Rahmam, M.M. "Modified solutions of some oscillators by iteration procedure", Journal of the Egyptian Mathematical Society, 21, pp. 68-73 (2013).

15. Wu, B.S., Sun, W.P., and Lim, C.W. "An analytical approximate technique for a class of strongly nonlinear oscillators", International Journal of Non-Linear Mechanics, 41, pp. 766-774 (2006).

16. Alam, M.S., Haque, M.E., and Hossian, M.B. "A new analytical technique to find periodic solutions of nonlinear systems", International Journal of NonLinear Mechanics, 42, pp. 1035-1045 (2007).

17. Hosen, M.A., Rahman, M.S., Alam, M.S., and Amin, M.R. "A new analytical technique for solving a class of strongly nonlinear conservative systems", Applied Mathematics and Computation, 218, pp. 5474-5486 (2012). 
18. He, J.H. "Variational approach for nonlinear oscillators", Chaos, Solitions \& Fractals, 34(5), pp. 14301439 (2007).

19. Khan, Y., Faraz, N., and Yildirim, A. "New soliton solutions of the generalized Zakharov equations using He's variational approach", Applied Mathematics Letters, 24(6), pp. 965-968 (2011).

20. Khan, Y., Akbarzade, M., and Kargar, A. "Coupling of homotopy and the variational approach for a conservative oscillator with strong odd-nonlinearity", Scientia Iranica A, 19(3), pp. 417-422 (2012).

21. He, J.H. "Preliminary report on the energy balance for nonlinear oscillators", Mechanics Research Communications, 29, pp. 107-111 ( 2002).

22. Zhang, H.L. "Periodic solutions for some strongly nonlinear oscillators by He's energy balance method", Computers \& Mathematics with Applications, 58, pp. 2480-2485 (2009).

23. Durmaz, S., Demirbag, S.A., and Kaya, M.O. "Highorder energy balance method based on collocation method", International Journal of Nonlinear Sciences and Numerical Simulation, 11, pp. 1-5 (2010).

24. Durmaz, S. and Kaya, M.O. "High-order energy balance method to nonlinear oscillators", Journal of Applied Mathematics, pp. 1-7 (2012).

25. Xie, F. and Gao, X. "Exact travelling wave solutions for a class of nonlinear partial differential equations", Chaos, Solitions \& Fractals, 19, pp. 1113-1117 (2004).

26. Lai, S.K., Lim, C.W., Wu, B.S., Wang, C., Zeng, Q.C., and He, X.F. "Newton-harmonic balancing approach for accurate solutions to nonlinear cubic-quintic
Duffing oscillators", Applied Mathematical Modelling, 33(2), pp. 852-866 (2009).

27. Guo, Z., Leung, A.Y.T., and Yang, H.X. "Iterative homotopy harmonic balancing approach for conservative oscillator with strong odd-nonlinearity", Applied Mathematical Modelling, 35, pp. 1717-1728 (2011).

28. Razzak, M.A. and Rahman, M.M. "Application of new novel energy balance method to strongly nonlinear oscillator systems", Results in Physics, 5, pp. 304-308 (2015).

29. Khan, Y. and Mirzabeigy, A. "Improved accuracy of He's energy balance method for analysis of conservative nonlinear oscillator", Neural Computing and Applications, 25, pp. 889-895 (2014).

30. Razzak, M.A. "An analytical approximate technique for solving cubic-quintic Duffing oscillator", Alexander Engineering Journal, 55, pp. 2959-2965 (2015).

\section{Biography}

Md. Abdur Razzak completed his MSc and BSc degrees at the Department of Applied Mathematics from Rajshahi University, Bangladesh. At present, he is an Assistant Professor at the Department of Mathematics, Rajshahi University of Engineering and Technology (RUET), and an MPhil fellow of RUET, Bangladesh. He is a lifetime member of Bangladesh Mathematical Society, and is the author of nine research articles in Mathematics. His research area is nonlinear dynamics. 